\title{
B-modes in cosmic shear from source redshift clustering
}

\author{
P. Schneider ${ }^{1,2}$, L. van Waerbeke ${ }^{3,4}$, and Y. Mellier ${ }^{3,5}$ \\ 1 Institut f. Astrophysik u. Extr. Forschung, Universität Bonn, Auf dem Hügel 71, 53121 Bonn, Germany \\ e-mail: peter@astro.uni-bonn.de \\ 2 Max-Planck-Institut f. Astrophysik, Postfach 1317, 85741 Garching, Germany \\ 3 Institute d'Astrophysique de Paris, 98 bis, boulevard Arago, 75014 Paris, France \\ 4 Canadian Institute for Theoretical Astrophysics, 60 St Georges Str., Toronto, M5S 3H8 Ontario, Canada \\ 5 Observatoire de Paris, DEMIRM/LERMA, 61 avenue de l'Observatoire, 75014 Paris, France
}

Received 19 December 2001 / Accepted 19 April 2002

\begin{abstract}
Weak gravitational lensing by the large scale structure can be used to probe the dark matter distribution in the Universe directly and thus to probe cosmological models. The recent detection of cosmic shear by several groups has demonstrated the feasibility of this new mode of observational cosmology. In the currently most extensive analysis of cosmic shear, it was found that the shear field contains unexpected modes, so-called B-modes, which are thought to be unaccountable for by lensing. B-modes can in principle be generated by an intrinsic alignment of galaxies from which the shear is measured, or may signify some remaining systematics in the data reduction and analysis. In this paper we show that B-modes in fact are produced by lensing itself. The effect comes about through the clustering of source galaxies, which in particular implies an angular separation-dependent clustering in redshift. After presenting the theory of the decomposition of a general shear field into E- and B-modes, we calculate their respective power spectra and correlation functions for a clustered source distribution. Numerical and analytical estimates of the relative strength of these two modes show that the resulting B-mode is very small on angular scales larger than a few arcminutes, but its relative contribution rises quickly towards smaller angular scales, with comparable power in both modes at a few arcseconds. The relevance of this effect with regard to the current cosmic shear surveys is discussed; it can not account for the apparent detection of a B-mode contribution on large angular scales in the cosmic shear analysis of van Waerbeke et al. (2002).
\end{abstract}

Key words. cosmology - gravitational lensing - large-scale structure of the Universe

\section{Introduction}

Gravitational lensing by the large-scale structure (LSS) leads to the distortion of the images of distant galaxies, owing to the tidal gravitational field of the matter inhomogeneities. Following very early work on the study of light propagation in an inhomogeneous universe (e.g., Gunn 1967; Kantowski 1969), Blandford et al. (1991), Miralda-Escude (1991) and Kaiser (1992) have pointed out that the observation of this "cosmic shear" effect immediately yields information about the statistical properties of the LSS and, thus, on cosmology. Non-linear evolution of the matter spectrum was taken into account in later analytical (e.g., Jain \& Seljak 1997; Bernardeau et al. 1997; Kaiser 1998; Schneider et al. 1998, hereafter SvWJK) and numerical (e.g., van Waerbeke et al. 1999; Jain et al. 2000; White \& Hu 2000) studies; see Mellier (1999) and Bartelmann \& Schneider (2001; hereafter BS01) for recent reviews.

It was only in 2000 when four teams nearly simultaneously and independently announced the first detections

Send offprint requests to: P. Schneider, e-mail: peter@astro.uni-bonn.de of cosmic shear from wide-field imaging data (Bacon et al. 2000; Kaiser et al. 2000; van Waerbeke et al. 2000; Wittman et al. 2000). The detections reported in these papers (and in Maoli et al. 2001, using the VLT, and Rhodes et al. 2001, using HST images obtained with the WFPC2 camera) concerned various two-point statistics, like the shear dispersion in an aperture, or the shear correlation function. In van Waerbeke et al. (2001), the aforementioned statistics, as well as the aperture mass statistics (SvWJK), were inferred from the effective 6.5 square degrees of high-quality imaging data. Very recently, Hämmerle et al. (2002) reported on a cosmic shear detection using HST parallel images taken with the STIS instrument on an effective angular scale of $\sim 30^{\prime \prime}$.

The shear field, originating from the inhomogeneous matter distribution, is a two-dimensional quantity, whereas the projected density field of the matter is a scalar field. The relation between the shear $\gamma(\boldsymbol{\theta})=\gamma_{1}(\boldsymbol{\theta})+\mathrm{i} \gamma_{2}(\boldsymbol{\theta})$ and the projected matter density $\kappa(\boldsymbol{\theta})$ is

$\gamma(\boldsymbol{\theta})=\frac{1}{\pi} \int_{\mathbb{R}^{2}} \mathrm{~d}^{2} \theta^{\prime} \mathcal{D}\left(\boldsymbol{\theta}-\boldsymbol{\theta}^{\prime}\right) \kappa\left(\boldsymbol{\theta}^{\prime}\right)$, 
with the kernel

$\mathcal{D}(\boldsymbol{\theta})=\frac{\theta_{2}^{2}-\theta_{1}^{2}-2 \mathrm{i} \theta_{1} \theta_{2}}{|\boldsymbol{\theta}|^{4}} ;$

here, $\kappa$ is the dimensionless surface mass density, i.e., the physical surface mass density divided by the "critical" surface mass density, as usual in gravitational lensing; we follow the notation of BS01 in this paper. Since the two shear components originate from a single scalar field, they are related to each other; in particular, their partial derivatives should satisfy compatability relations, as we shall discuss in Sect. 2 below. In analogy with the polarization of the CMB, a shear field satisfying these compatability relations is called an E-mode shear field.

Pen et al. (2002) pointed out that the cosmic shear data of van Waerbeke et al. (2001) contains not only an E-mode, but also a statistically significant B-mode contribution in addition. Such B-modes can be generated by effects unrelated to gravitational lensing, such as intrinsic alignment of galaxies (e.g., Heavens et al. 2000; Crittenden et al. 2001a; Croft \& Metzler 2000; Catelan et al. 2000) or remaining systematics in the data reduction and analysis.

In this paper we show that a B-mode contribution to the cosmic shear is obtained by lensing itself. A B-mode is generated owing to the clustering properties of the faint galaxies from which the shear is measured. This spatial clustering implies an angular separation-dependent clustering in redshift, which is the origin not only of the B-mode of the shear, but also of an additional E-mode contribution.

The paper is organized as follows: in Sect. 2 we provide a tutorial description of the E/B-mode decomposition of a shear field. Most of the results there were derived before in Crittenden et al. (2001b, hereafter C01), but we formulate them in standard lensing notation, which will be needed for the later investigation. The calculation of two-point cosmic shear statistics in the presence of source clustering is presented in Sect. 3 where it is shown that this clustering produces a B-mode. Numerical and analytical estimates of the amplitude of this B-mode are provided in Sect. 4 and discussed in Sect. 5 .

\section{E/B-mode decomposition of a shear field}

In this section we provide the basic relations for the decomposition of the shear field into E- and B-modes. Most of these relations have been obtained in $\mathrm{C} 01$; we shall write them here in standard lensing notation.

\subsection{Motivation}

If the shear field is obtained from a projected surface mass density $\kappa$ as in Eq. (1), then the gradient of the density field $\kappa$ is related to the first spatial derivatives of the shear components in the following way (Kaiser 1995):

$$
\nabla \kappa=\left(\begin{array}{c}
\gamma_{1,1}+\gamma_{2,2} \\
\gamma_{2,1}-\gamma_{1,2}
\end{array}\right) \equiv \boldsymbol{u}
$$

The vector field $\boldsymbol{u}$ can be obtained from observations, e.g. in weak lensing cluster mass reconstructions, by obtaining a smoothed version of the shear field and then differentiating this numerically. Owing to noise, the resulting ("observed") field $\boldsymbol{u}$ will in general not be a gradient field. The non-gradient part of $\boldsymbol{u}$ is then a readily identifiable noise component and can be filtered out in the mass reconstruction. Seitz \& Schneider (1996) provided a scheme for this noise filtering (see also Seitz \& Schneider 2001 for a simpler though equivalent method), which was shown by Lombardi \& Bertin (1998) to be an optimal reconstruction method.

If the shear field cannot be ascribed to a single geometrically thin gravitational lens, the non-gradient part of $\boldsymbol{u}$ is not necessarily due to noise. For example, if the galaxies have intrinsic alignments, this may induce a curlpart of $\boldsymbol{u}$. To project out the gradient and curl part of $\boldsymbol{u}$, we take a further derivative of $\boldsymbol{u}$, and define

$\nabla^{2} \kappa^{\mathrm{E}}=\nabla \cdot \boldsymbol{u} ; \quad \nabla^{2} \kappa^{\mathrm{B}}=\nabla \times \boldsymbol{u} \equiv u_{2,1}-u_{1,2}$.

Through these relations, $\kappa^{\mathrm{E}}$ and $\kappa^{\mathrm{B}}$ are not uniquely defined on a finite data field; as discussed in Seitz \& Schneider (1996), a further condition is needed to specify the two modes uniquely. However, we shall not be concerned here with finite-field effects.

An alternative way to define $\kappa^{\mathrm{E}}$ and $\kappa^{\mathrm{B}}$ is through the Kaiser \& Squires (1993) mass-reconstruction relation

$\kappa^{\mathrm{E}}(\boldsymbol{\theta})+\mathrm{i} \kappa^{\mathrm{B}}(\boldsymbol{\theta})=\frac{1}{\pi} \int_{\mathbb{R}^{2}} \mathrm{~d}^{2} \theta^{\prime} \mathcal{D}^{*}\left(\boldsymbol{\theta}-\boldsymbol{\theta}^{\prime}\right) \gamma\left(\boldsymbol{\theta}^{\prime}\right)$,

which formally requires data on an infinite field; here, $\mathcal{D}^{*}$ denotes the complex-conjugate of the complex kernel (2). If $\gamma$ is of the form (1) with a real field $\kappa$, then the result from (5) will be real, $\kappa^{\mathrm{E}}=\kappa, \kappa^{\mathrm{B}}=0$. In applications of the KS-formula (5) to observational data, where the recovered shear field necessarily is noisy, one usually takes the real part of the integral to obtain the projected mass density field. For a general shear field, the result from (5) will be complex, with the real part yielding the E-mode, and the imaginary part corresponding to the B-mode.

To simplify notation and calculations, it is convenient to express two-component quantities in terms of complex numbers. We define the E- and B-mode potentials $\psi^{\mathrm{E}}$ and $\psi^{\mathrm{B}}$ by

$\nabla^{2} \psi^{\mathrm{E}, \mathrm{B}}=2 \kappa^{\mathrm{E}, \mathrm{B}}$,

and combine the two modes into the complex fields

$\kappa=\kappa^{\mathrm{E}}+\mathrm{i} \kappa^{\mathrm{B}}, \quad \psi=\psi^{\mathrm{E}}+\mathrm{i} \psi^{\mathrm{B}}$.

The complex shear $\gamma=\gamma_{1}+\mathrm{i} \gamma_{2}$ is obtained from the potential $\psi$ by $\gamma=D \psi$, where the differential operator $D=\left(\partial_{11}-\partial_{22}\right) / 2+\mathrm{i} \partial_{12} ;$ hence,

$\gamma=\left[\frac{1}{2}\left(\psi_{, 11}^{\mathrm{E}}-\psi_{, 22}^{\mathrm{E}}\right)-\psi_{, 12}^{\mathrm{B}}\right]+\mathrm{i}\left[\psi_{, 12}^{\mathrm{E}}+\frac{1}{2}\left(\psi_{, 11}^{\mathrm{B}}-\psi_{, 22}^{\mathrm{B}}\right)\right]$.

Inserting this into (3) yields

$\boldsymbol{u}=\left(\begin{array}{c}\kappa_{, 1}^{\mathrm{E}}-\kappa_{, 2}^{\mathrm{B}} \\ \kappa_{, 2}^{\mathrm{E}}+\kappa_{, 1}^{\mathrm{B}}\end{array}\right)$. 
Indeed, the shear field can be decomposed into E/B-modes, $\gamma=\gamma^{\mathrm{E}}+\mathrm{i} \gamma^{\mathrm{B}}$, with

$\gamma^{\mathrm{E}}=\frac{\mathcal{D}}{\pi} * \mathcal{R e}\left[\frac{\mathcal{D}^{*}}{\pi} * \gamma\right]$

$\gamma^{\mathrm{B}}=\frac{\mathcal{D}}{\pi} * \mathcal{I} \mathrm{m}\left[\frac{\mathcal{D}^{*}}{\pi} * \gamma\right]$,

where the operator $\mathcal{D}$ is defined in Eqs. (1), (2), and “*” denotes complex conjugation. Thus, the two components can be obtained from the shear field by filtering, except for an additive constant.

\subsection{Shear correlation functions and power spectra}

The discussion above dealt with the shear field itself. In the application to cosmic shear, one usually does not investigate the shear of a $\kappa$-field itself, but its statistical properties. In this paper we shall concentrate solely on two-point statistical measures of the cosmic shear, and their decomposition into E- and B-modes.

Owing to statistical homogeneity and isotropy of the Universe, $\kappa^{\mathrm{E}, \mathrm{B}}(\boldsymbol{\theta})$ are homogeneous and isotropic random fields. Hence, in terms of their Fourier transforms

$\hat{\kappa}^{\mathrm{E}, \mathrm{B}}(\boldsymbol{\ell})=\int \mathrm{d}^{2} \theta \mathrm{e}^{\mathrm{i} \boldsymbol{\ell} \cdot \boldsymbol{\theta}} \kappa^{\mathrm{E}, \mathrm{B}}(\boldsymbol{\theta})$,

one defines the two power spectra $P_{\mathrm{E}}, P_{\mathrm{B}}$, and the cross power spectrum $P_{\mathrm{EB}}$ by

$\left\langle\hat{\kappa}^{\mathrm{E}}(\boldsymbol{\ell}) \hat{\kappa}^{\mathrm{E} *}\left(\boldsymbol{\ell}^{\prime}\right)\right\rangle=(2 \pi)^{2} \delta_{\mathrm{D}}\left(\boldsymbol{\ell}-\boldsymbol{\ell}^{\prime}\right) P_{\mathrm{E}}(\ell)$,

$\left\langle\hat{\kappa}^{\mathrm{B}}(\boldsymbol{\ell}) \hat{\kappa}^{\mathrm{B} *}\left(\ell^{\prime}\right)\right\rangle=(2 \pi)^{2} \delta_{\mathrm{D}}\left(\boldsymbol{\ell}-\boldsymbol{\ell}^{\prime}\right) P_{\mathrm{B}}(\ell)$,

$\left\langle\hat{\kappa}^{\mathrm{E}}(\boldsymbol{\ell}) \hat{\kappa}^{\mathrm{B} *}\left(\boldsymbol{\ell}^{\prime}\right)\right\rangle=(2 \pi)^{2} \delta_{\mathrm{D}}\left(\boldsymbol{\ell}-\boldsymbol{\ell}^{\prime}\right) P_{\mathrm{EB}}(\ell)$,

where $\delta_{\mathrm{D}}$ denotes Dirac's delta distribution. In terms of the complex field $\kappa$, we then have

$\left\langle\hat{\kappa}(\ell) \hat{\kappa}^{*}\left(\boldsymbol{\ell}^{\prime}\right)\right\rangle=(2 \pi)^{2} \delta_{\mathrm{D}}\left(\boldsymbol{\ell}-\boldsymbol{\ell}^{\prime}\right)\left[P_{\mathrm{E}}(\ell)+P_{\mathrm{B}}(\ell)\right]$,

$\left\langle\hat{\kappa}(\boldsymbol{\ell}) \hat{\kappa}\left(\boldsymbol{\ell}^{\prime}\right)\right\rangle=(2 \pi)^{2} \delta_{\mathrm{D}}\left(\boldsymbol{\ell}+\boldsymbol{\ell}^{\prime}\right)$

$$
\times\left[P_{\mathrm{E}}(\ell)-P_{\mathrm{B}}(\ell)+2 \mathrm{i} P_{\mathrm{EB}}(\ell)\right] .
$$

The Fourier transform $\hat{\gamma}(\ell)$ of the shear is related to $\hat{\kappa}(\ell)$ through

$\hat{\gamma}(\ell)=\left(\frac{\ell_{1}^{2}-\ell_{2}^{2}+2 \mathrm{i} \ell_{1} \ell_{2}}{|\ell|^{2}}\right) \hat{\kappa}(\ell)=\mathrm{e}^{2 \mathrm{i} \beta} \hat{\kappa}(\ell)$,

where $\beta$ is the polar angle of $\boldsymbol{\ell}$. The correlators of the shear then become

$$
\begin{aligned}
\left\langle\hat{\gamma}(\ell) \hat{\gamma}^{*}\left(\ell^{\prime}\right)\right\rangle= & (2 \pi)^{2} \delta_{\mathrm{D}}\left(\boldsymbol{\ell}-\boldsymbol{\ell}^{\prime}\right)\left[P_{\mathrm{E}}(\ell)+P_{\mathrm{B}}(\ell)\right], \\
\left\langle\hat{\gamma}(\ell) \hat{\gamma}\left(\ell^{\prime}\right)\right\rangle= & (2 \pi)^{2} \delta_{\mathrm{D}}\left(\boldsymbol{\ell}+\boldsymbol{\ell}^{\prime}\right) \mathrm{e}^{4 \mathrm{i} \beta} \\
& \times\left[P_{\mathrm{E}}(\ell)-P_{\mathrm{B}}(\ell)+2 \mathrm{i} P_{\mathrm{EB}}(\ell)\right] .
\end{aligned}
$$

Next we define the correlation functions of the shear. This is done by considering pairs of positions $\boldsymbol{\vartheta}$ and $\boldsymbol{\theta}+\boldsymbol{\vartheta}$, and defining the tangential and cross-component of the shear at position $\boldsymbol{\vartheta}$ for this pair as

$\gamma_{\mathrm{t}}=-\mathcal{R} \mathrm{e}\left(\gamma \mathrm{e}^{-2 \mathrm{i} \varphi}\right), \quad \gamma_{\times}=-\mathcal{I} \mathrm{m}\left(\gamma \mathrm{e}^{-2 \mathrm{i} \varphi}\right)$,

respectively, where $\varphi$ is the polar angle of the separation vector $\boldsymbol{\theta}$. Then, the shear correlation functions are defined as

$$
\begin{aligned}
& \xi_{+}(\theta)=\left\langle\gamma_{\mathrm{t}} \gamma_{\mathrm{t}}\right\rangle+\left\langle\gamma_{\times} \gamma_{\times}\right\rangle(\theta), \\
& \xi_{-}(\theta)=\left\langle\gamma_{\mathrm{t}} \gamma_{\mathrm{t}}\right\rangle-\left\langle\gamma_{\times} \gamma_{\times}\right\rangle(\theta), \\
& \xi_{\times}(\theta)=\left\langle\gamma_{\mathrm{t}} \gamma_{\times}\right\rangle(\theta)
\end{aligned}
$$

The shear correlation functions are most easily calculated by choosing $\boldsymbol{\theta}=(\theta, 0)$, in which case $\gamma_{\mathrm{t}}=-\gamma_{1}, \gamma_{\times}=-\gamma_{2}$, and expressing the shear in terms of its Fourier modes,

$$
\begin{aligned}
\left\langle\gamma(\mathbf{0}) \gamma^{*}(\boldsymbol{\theta})\right\rangle & =\xi_{+}(\theta) \\
& =\int \frac{\mathrm{d}^{2} \ell}{(2 \pi)^{2}} \int \frac{\mathrm{d}^{2} \ell^{\prime}}{(2 \pi)^{2}} \mathrm{e}^{\mathrm{i} \ell^{\prime} \cdot \boldsymbol{\theta}}\left\langle\hat{\gamma}(\ell) \hat{\gamma}^{*}\left(\boldsymbol{\ell}^{\prime}\right)\right\rangle \\
& =\int_{0}^{\infty} \frac{\mathrm{d} \ell \ell}{2 \pi} J_{0}(\ell \theta)\left[P_{\mathrm{E}}(\ell)+P_{\mathrm{B}}(\ell)\right] \\
\langle\gamma(\mathbf{0}) \gamma(\boldsymbol{\theta})\rangle & =\xi_{-}(\theta)+2 \mathrm{i} \xi_{\times}(\theta) \\
& =\int \frac{\mathrm{d}^{2} \ell}{(2 \pi)^{2}} \int \frac{\mathrm{d}^{2} \ell^{\prime}}{(2 \pi)^{2}} \mathrm{e}^{-\mathrm{i} \ell^{\prime} \cdot \boldsymbol{\theta}}\left\langle\hat{\gamma}(\boldsymbol{\ell}) \hat{\gamma}\left(\boldsymbol{\ell}^{\prime}\right)\right\rangle \\
& =\int_{0}^{\infty} \frac{\mathrm{d} \ell \ell}{2 \pi} J_{4}(\ell \theta)\left[P_{\mathrm{E}}(\ell)-P_{\mathrm{B}}(\ell)+2 \mathrm{i} P_{\mathrm{EB}}(\ell)\right]
\end{aligned}
$$

Making use of the orthogonality of Bessel functions,

$\int_{0}^{\infty} \mathrm{d} \theta \theta J_{\nu}(s \theta) J_{\nu}(t \theta)=\frac{\delta_{\mathrm{D}}(s-t)}{t}$

we can invert the relations (15) and (16) and express the power spectra in terms of the correlation functions,

$$
\begin{aligned}
& P_{\mathrm{E}}(\ell)=\pi \int_{0}^{\infty} \mathrm{d} \theta \theta\left[\xi_{+}(\theta) J_{0}(\ell \theta)+\xi_{-}(\theta) J_{4}(\ell \theta)\right], \\
& P_{\mathrm{B}}(\ell)=\pi \int_{0}^{\infty} \mathrm{d} \theta \theta\left[\xi_{+}(\theta) J_{0}(\ell \theta)-\xi_{-}(\theta) J_{4}(\ell \theta)\right], \\
& P_{\mathrm{EB}}(\ell)=2 \pi \int_{0}^{\infty} \mathrm{d} \theta \theta \xi_{\times}(\theta) J_{4}(\ell \theta) .
\end{aligned}
$$

Hence, we have now expressed the various power spectra in terms of the directly observable correlation functions $\xi$. One notes that the correlation functions $\xi_{+}$and $\xi_{-}$depend on both, the E- and B-mode power spectra, whereas the cross-correlation $\xi_{\times}$depends on the cross-power $P_{\mathrm{EB}}$ only. It is obvious that the cross-power and its corresponding correlation function do not "mix in" with the E- and B-mode; in addition, the cross-power vanishes if the shear field is statistically invariant under parity transformations, which leave $\gamma_{\mathrm{t}}$ unchanged, but transform $\gamma_{\times} \rightarrow-\gamma_{\times}$. One can therefore assume that in realistic cases, $\xi_{\times} \equiv 0 \equiv P_{\mathrm{EB}}$. However, since cosmic shear is measured from finite data 
fields, cosmic variance may lead to a non-zero measurement of the cross-power; in fact, the measurement of the cross-power may serve as a lower limit on error bars of the other power spectra.

For a determination of the power spectra, the expressions (18) require a measurement of the correlation functions over an infinite range in angle; whereas the correlation functions decrease with $\theta$ and become very small for large $\theta$, so that in effect the integrals can be replaced by ones over a finite range of integration, one might want to obtain more local decompositions into E- and B-modes.

\subsection{E/B-mode correlation functions}

We define the four correlation functions

$\xi_{\mathrm{E}, \mathrm{B}+}(\theta)=\int \frac{\mathrm{d} \ell \ell}{2 \pi} P_{\mathrm{E}, \mathrm{B}}(\ell) J_{0}(\theta \ell)$

$\xi_{\mathrm{E}, \mathrm{B}-}(\theta)=\int \frac{\mathrm{d} \ell \ell}{2 \pi} P_{\mathrm{E}, \mathrm{B}}(\ell) J_{4}(\theta \ell)$

which are defined such that in the absence of B-modes, $\xi_{\mathrm{E} \pm} \equiv \xi_{ \pm} ;$these four correlation functions have also been defined in $\mathrm{C} 01$, although only the "+" ones were investigated in more detail there. Inserting (18) into the foregoing definitions, one obtains

$$
\begin{aligned}
\xi_{\mathrm{E}+}(\theta)= & \frac{1}{2} \int_{0}^{\infty} \mathrm{d} \ell \ell \int_{0}^{\infty} \mathrm{d} \vartheta \vartheta J_{0}(\theta \ell) \\
& \times\left[\xi_{+}(\vartheta) J_{0}(\vartheta \ell)+\xi_{-}(\vartheta) J_{4}(\vartheta \ell)\right] .
\end{aligned}
$$

The $\ell$-integration can be carried out; consider the function

$$
G(\vartheta, \theta)=\int_{0}^{\infty} \mathrm{d} t t J_{0}(t \vartheta) J_{4}(t \theta)
$$

Making use of the recurrence relations for Bessel functions, one can express $J_{4}$ as

$$
J_{4}(x)=\frac{24}{x^{2}} J_{2}(x)-\frac{8}{x} J_{1}(x)+J_{0}(x)
$$

By using Eq. (11.4.41) of Abramowitz \& Stegun (1965), together with (17), one can perform the integration in (21) term by term to obtain

$G(\vartheta, \theta)=\left(\frac{4}{\theta^{2}}-\frac{12 \vartheta^{2}}{\theta^{4}}\right) \mathrm{H}(\theta-\vartheta)+\frac{1}{\theta} \delta_{\mathrm{D}}(\theta-\vartheta)$,

where $\mathrm{H}(x)$ is the Heaviside step function. We also note the interesting property,

$$
\begin{aligned}
\int_{0}^{\infty} \mathrm{d} \vartheta \vartheta G(\vartheta, \theta) G(\vartheta, \varphi) & =\frac{\delta_{\mathrm{D}}(\theta-\varphi)}{\varphi} \\
& =\int_{0}^{\infty} \mathrm{d} \vartheta \vartheta G(\theta, \vartheta) G(\varphi, \vartheta)
\end{aligned}
$$

which is readily shown using (17). Thus, (20) becomes

$$
\begin{aligned}
\xi_{\mathrm{E}+}(\theta) & =\frac{1}{2}\left[\xi_{+}(\theta)+\int_{0}^{\infty} \mathrm{d} \vartheta \vartheta \xi_{-}(\vartheta) G(\theta, \vartheta)\right] \\
& =\frac{1}{2}\left[\xi_{+}(\theta)+\xi_{-}(\theta)+\int_{\theta}^{\infty} \frac{\mathrm{d} \vartheta}{\vartheta} \xi_{-}(\vartheta)\left(4-12 \frac{\theta^{2}}{\vartheta^{2}}\right)\right]
\end{aligned}
$$

We have obtained a combination of shear correlation functions which depends only on the E-modes; however, in order to obtain $\xi_{\mathrm{E}+}$ one would need to know $\xi_{-}$for arbitrarily large separations. However,

$$
\begin{aligned}
\xi_{\mathrm{E}-}(\theta) & =\frac{1}{2}\left[\xi_{-}(\theta)+\int_{0}^{\infty} \mathrm{d} \vartheta \vartheta \xi_{+}(\vartheta) G(\vartheta, \theta)\right] \\
& =\frac{1}{2}\left[\xi_{-}(\theta)+\xi_{+}(\theta)+\int_{0}^{\theta} \frac{\mathrm{d} \vartheta \vartheta}{\theta^{2}} \xi_{+}(\vartheta)\left(4-12 \frac{\vartheta^{2}}{\theta^{2}}\right)\right]
\end{aligned}
$$

depends on the observable correlation functions $\xi_{ \pm}$over a finite range only and thus can be measured from finite data sets. Analogously, we find for the B-mode correlation functions

$$
\begin{aligned}
\xi_{\mathrm{B}+}(\theta) & =\frac{1}{2}\left[\xi_{+}(\theta)-\int_{0}^{\infty} \mathrm{d} \vartheta \vartheta \xi_{-}(\vartheta) G(\theta, \vartheta)\right] \\
& =\frac{1}{2}\left[\xi_{+}(\theta)-\xi_{-}(\theta)-\int_{\theta}^{\infty} \frac{\mathrm{d} \vartheta}{\vartheta} \xi_{-}(\vartheta)\left(4-12 \frac{\theta^{2}}{\vartheta^{2}}\right)\right] \\
\xi_{\mathrm{B}-}(\theta) & =\frac{1}{2}\left[-\xi_{-}(\theta)+\int_{0}^{\infty} \mathrm{d} \vartheta \vartheta \xi_{+}(\vartheta) G(\vartheta, \theta)\right] \\
& =\frac{1}{2}\left[\xi_{+}(\theta)-\xi_{-}(\theta)+\int_{0}^{\theta} \frac{\mathrm{d} \vartheta \vartheta}{\theta^{2}} \xi_{+}(\vartheta)\left(4-12 \frac{\vartheta^{2}}{\theta^{2}}\right)\right]
\end{aligned}
$$

We note that $\xi_{\mathrm{E}+}+\xi_{\mathrm{B}+}=\xi_{+}, \xi_{\mathrm{E}-}-\xi_{\mathrm{B}-}=\xi_{-}$. In order to calculate the E- and B-mode correlation functions, one needs to know either the observable correlation function $\xi_{-}$to arbitrarily large, or $\xi_{+}$to arbitrarily small separations. This is of course impossible, owing to the finite size of data fields on the one hand, and the impossibility to measure shapes of very close pairs of galaxies. In either case, the lack of measurements for large (or small) separations can be summarized in two constants: suppose that $\xi_{+}$can be measured down to separations of $\theta_{\min }$; then, the integral in the "-" modes over $\xi_{+}$can be split into one from 0 to $\theta_{\min }$, and one from $\theta_{\min }$ to $\theta$. The former one has the $\theta$-dependence $a / \theta^{2}-b / \theta^{4}$, where $a, b$ are two constants, depending on $\xi_{+}$for $\theta<\theta_{\min }$. The decline of this contribution, with leading order $\theta^{-2}$, shows that it has a small influence on the determination of the "-" modes for $\theta \gg \theta_{\min }$; in addition, "reasonable guesses" for $a$ and $b$ may be obtained by extrapolating the measured $\xi_{+}$towards small angles. The same reasoning shows the analogous situation for the " + " modes.

\subsection{Aperture measures}

One very convenient way to separate E- and B-modes is provided by the aperture mass: defining the tangential and cross component of the shear relative to the center of a circular aperture of angular radius $\theta$, and defining

$$
\begin{aligned}
M_{\mathrm{ap}}(\theta) & =\int \mathrm{d}^{2} \vartheta Q(|\boldsymbol{\vartheta}|) \gamma_{\mathrm{t}}(\boldsymbol{\vartheta}), \\
M_{\perp}(\theta) & =\int \mathrm{d}^{2} \vartheta Q(|\boldsymbol{\vartheta}|) \gamma_{\times}(\boldsymbol{\vartheta}),
\end{aligned}
$$


it was shown in C01 that E-modes do not contribute to $M_{\perp}$, and B-modes do not contribute to $M_{\mathrm{ap}}$; in fact, this can be easily seen directly by inserting the Fourier transform of the shear (11) into (27). Here, $Q(\vartheta)$ is an axially-symmetric weight function which can be chosen arbitrarily. The integration range in the foregoing equations extends over the support of the weight function $Q$. The aperture mass was introduced by Schneider (1996) in an attempt to detect mass peaks from shear fields, and later used by SvWJK as a two- and three-point statistics for cosmic shear. $M_{\text {ap }}$ can also be written as a filtered version of the surface mass density (with a different and compensated weight function which is related to $Q$ ), whereas $M_{\perp}$ has no direct physical interpretation; in the absence of B-modes, $M_{\perp}$ should vanish, and any non-vanishing signal is usually interpreted as being due to noise or remaining systematics, and thus as a convenient error estimate for $M_{\mathrm{ap}}$. In SvWJK, a family of convenient weight functions $Q$ was considered, the simplest of which is

$Q(\vartheta)=\frac{6}{\pi \theta^{2}} \frac{\vartheta^{2}}{\theta^{2}}\left(1-\frac{\vartheta^{2}}{\theta^{2}}\right) \mathrm{H}(\theta-\vartheta)$,

where $\theta$ is the radius of the aperture. This form of the weight function shall be assumed in the following.

Using the complex number

$$
\begin{aligned}
M(\theta) & =M_{\mathrm{ap}}(\theta)+\mathrm{i} M_{\perp}(\theta) \\
& =-\int \mathrm{d}^{2} \vartheta Q(|\boldsymbol{\vartheta}|) \gamma(\boldsymbol{\vartheta}) \mathrm{e}^{-2 \mathrm{i} \varphi},
\end{aligned}
$$

where $\varphi$ is the polar angle of $\boldsymbol{\vartheta}$, one finds that

$$
\begin{aligned}
\left\langle M_{\mathrm{ap}}^{2}\right\rangle+\left\langle M_{\perp}^{2}\right\rangle & =\left\langle M M^{*}\right\rangle \\
& =\frac{1}{2 \pi} \int_{0}^{\infty} \mathrm{d} \ell \ell\left[P_{\mathrm{E}}(\ell)+P_{\mathrm{B}}(\ell)\right] W(\theta \ell),
\end{aligned}
$$

with

$W(\eta):=\frac{576 J_{4}^{2}(\eta)}{\eta^{4}}$,

which was derived by using the Fourier transform (11) of the shear, and the final steps are as in SvWJK. Similarly, one obtains

$$
\begin{aligned}
\left\langle M_{\mathrm{ap}}^{2}\right\rangle & -\left\langle M_{\perp}^{2}\right\rangle+2 \mathrm{i}\left\langle M_{\mathrm{ap}} M_{\perp}\right\rangle=\langle M M\rangle \\
= & \frac{1}{2 \pi} \int_{0}^{\infty} \mathrm{d} \ell \ell\left[P_{\mathrm{E}}(\ell)-P_{\mathrm{B}}(\ell)+2 \mathrm{i} P_{\mathrm{EB}}(\ell)\right] W(\theta \ell) .
\end{aligned}
$$

Combining the two previous equations, one thus gets

$$
\begin{aligned}
& \left\langle M_{\mathrm{ap}}^{2}\right\rangle(\theta)=\frac{1}{2 \pi} \int_{0}^{\infty} \mathrm{d} \ell \ell P_{\mathrm{E}}(\ell) W(\theta \ell), \\
& \left\langle M_{\perp}^{2}\right\rangle(\theta)=\frac{1}{2 \pi} \int_{0}^{\infty} \mathrm{d} \ell \ell P_{\mathrm{B}}(\ell) W(\theta \ell),
\end{aligned}
$$

so that these two-point statistics clearly separate E- and B-modes. In addition, they provide a highly localized measure of the corresponding power spectra, since the filter function $W(\eta)$ involved is quite narrow (see SvWJK); in fact, Bartelmann \& Schneider (1999) have shown that replacing $W(\eta)$ in $(31)$ by $A \delta_{\mathrm{D}}\left(\eta-\eta_{0}\right)$, with $\eta_{0} \approx 4.25$, provides a fairly accurate approximation. Furthermore, (30) can be used to check whether the shear data contain a contribution from the cross power $P_{\mathrm{EB}}$.

The aperture measures can be obtained directly from the observational data by laying down a grid of points, at each of which $M_{\mathrm{ap}}$ and $M_{\perp}$ are calculated from (27). However, obtaining the dispersion with this strategy turns out to be difficult in practice, since data fields usually contain holes and gaps, e.g. because of masking (for bright stars), bad columns etc. It is therefore interesting to calculate these dispersions directly in terms of the correlation functions, which can be done by inserting (18) into (31),

$$
\begin{aligned}
& \left\langle M_{\mathrm{ap}}^{2}\right\rangle(\theta)=\frac{1}{2} \int \frac{\mathrm{d} \vartheta \vartheta}{\theta^{2}}\left[\xi_{+}(\vartheta) T_{+}\left(\frac{\vartheta}{\theta}\right)+\xi_{-}(\vartheta) T_{-}\left(\frac{\vartheta}{\theta}\right)\right], \\
& \left\langle M_{\perp}^{2}\right\rangle(\theta)=\frac{1}{2} \int \frac{\mathrm{d} \vartheta \vartheta}{\theta^{2}}\left[\xi_{+}(\vartheta) T_{+}\left(\frac{\vartheta}{\theta}\right)-\xi_{-}(\vartheta) T_{-}\left(\frac{\vartheta}{\theta}\right)\right],
\end{aligned}
$$

where we have defined the functions

$$
\begin{aligned}
& T_{+}(x)=576 \int_{0}^{\infty} \frac{\mathrm{d} t}{t^{3}} J_{0}(x t)\left[J_{4}(t)\right]^{2}, \\
& T_{-}(x)=576 \int_{0}^{\infty} \frac{\mathrm{d} t}{t^{3}} J_{4}(x t)\left[J_{4}(t)\right]^{2} .
\end{aligned}
$$

The integration range in (32) formally extends from zero to infinity, but as we shall see shortly, the functions $T_{ \pm}(x)$ vanish for $x>2$, so the integration range is $0 \leq \vartheta \leq 2 \theta$ : for $T_{-}(x)$, an analytic expression can be obtained, using Eq. (6.578.9) of Gradshteyn \& Ryzhik (1980),

$T_{-}(x)=\frac{192}{35 \pi} x^{3}\left(1-\frac{x^{2}}{4}\right)^{7 / 2} \mathrm{H}(2-x)$,

so that $T_{-}(x)$ vanishes for $x>2$. Furthermore, the two functions $T_{+}$and $T_{-}(x)$ are related: using (17), one finds that

$\int_{0}^{\infty} \mathrm{d} x x T_{+}(x) J_{0}(t x)=W(t)=\int_{0}^{\infty} \mathrm{d} x x T_{-}(x) J_{4}(t x)$

so that

$T_{-}(x)=\int_{0}^{\infty} \mathrm{d} t t W(t) J_{4}(x t)=\int_{0}^{\infty} \mathrm{d} y y T_{+}(y) G(y, x)$
$T_{+}(x)=\int_{0}^{\infty} \mathrm{d} t t W(t) J_{0}(x t)=\int_{0}^{\infty} \mathrm{d} y y T_{-}(y) G(x, y)$

using the latter expression, together with (34) and (22), one obtains

$$
\begin{aligned}
T_{+}(x)= & \frac{6\left(2-15 x^{2}\right)}{5}\left[1-\frac{2}{\pi} \arcsin \left(\frac{x}{2}\right)\right] \\
& +\frac{x \sqrt{4-x^{2}}}{100 \pi}\left(120+2320 x^{2}-754 x^{4}+132 x^{6}-9 x^{8}\right)
\end{aligned}
$$




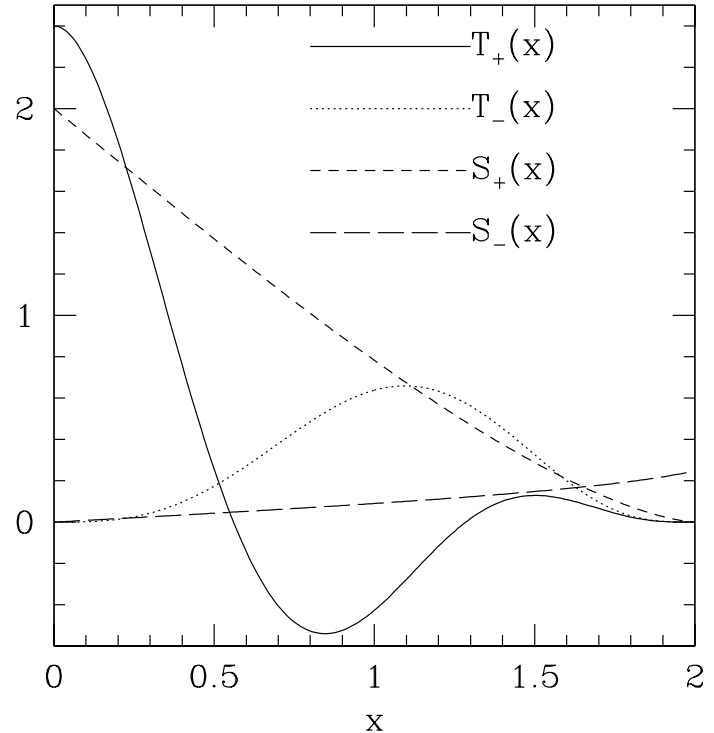

Fig. 1. The four functions defined in text.

for $x \leq 2$, and $T_{+}(x)$ vanishes for $x>2$. Hence, the integrals in (32) extend only over $0 \leq \vartheta \leq 2 \theta$, so that $\left\langle M_{\text {ap }}^{2}\right\rangle$ and $\left\langle M_{\perp}^{2}\right\rangle$ can be obtained directly in terms of the observable correlation function $\xi_{ \pm}$over a finite interval. The two functions $T_{ \pm}$are plotted in Fig. 1 .

\subsection{Shear dispersion}

Another cosmic shear statistics often employed is the shear dispersion in a circle of angular radius $\theta$. It is related to the power spectra by

$$
\left\langle|\bar{\gamma}|^{2}\right\rangle(\theta)=\frac{1}{2 \pi} \int \mathrm{d} \ell \ell\left(P_{\mathrm{E}}+P_{\mathrm{B}}\right)(\ell) W_{\mathrm{TH}}(\ell \theta),
$$

where

$$
W_{\mathrm{TH}}(\eta)=\frac{4 J_{1}^{2}(\eta)}{\eta^{2}}
$$

is the top-hat filter function. In contrast to the aperture measures of the previous subsection, the shear dispersion (36) contains both modes; furthermore, the filter function $W_{\mathrm{TH}}(\eta)$ is much broader than $W(\eta)$ in $(29)$, as demonstrated in SvWJK. It thus provides a much less localized measure of the power spectra than the aperture measures. On the other hand, this larger filter width implies that the signal of the shear dispersion is larger than that of the aperture measures, which explains why the first cosmic shear detections (van Waerbeke et al. 2000; Bacon et al. 2001; Kaiser et al. 2000) were obtained in terms of the shear dispersion.

As before, the shear dispersion can be obtained by calculating the mean shear in circles which are laid down on a grid of points, with the drawback of being affected by gaps in the data field. Alternatively, the shear dispersion can be obtained directly from the correlation function,

$\left\langle|\bar{\gamma}|^{2}\right\rangle(\theta)=\int \frac{\mathrm{d} \vartheta \vartheta}{\theta^{2}} \xi_{+}(\vartheta) S_{+}\left(\frac{\vartheta}{\theta}\right)$

where (van Waerbeke 2000)

$S_{+}(x)=\frac{1}{\pi}\left[4 \arccos \left(\frac{x}{2}\right)-x \sqrt{4-x^{2}}\right]$

for $x \leq 2$, and zero otherwise. Hence, the integral in (38) extends only over the finite interval $0 \leq \vartheta \leq 2 \theta$, which makes this a convenient way to calculate the shear dispersion.

One can also define the shear dispersions of the E- and B-mode, according to

$\left\langle|\bar{\gamma}|^{2}\right\rangle_{\mathrm{E}, \mathrm{B}}(\theta)=\frac{1}{2 \pi} \int \mathrm{d} \ell \ell P_{\mathrm{E}, \mathrm{B}}(\ell) W_{\mathrm{TH}}(\ell \theta)$,

but they cannot be individually obtained from measuring the shear directly. Nevertheless, both of these dispersions can be obtained in terms of the correlation functions,

$\left\langle|\bar{\gamma}|^{2}\right\rangle_{\mathrm{E}, \mathrm{B}}(\theta)=\int \frac{\mathrm{d} \vartheta \vartheta}{2 \theta^{2}}\left[\xi_{+}(\vartheta) S_{+}\left(\frac{\vartheta}{\theta}\right) \pm \xi_{-}(\vartheta) S_{-}\left(\frac{\vartheta}{\theta}\right)\right]$,

which can be derived in close analogy to the derivation of (32), and the function $S_{-}$is related to $S_{+}$in the same way as the corresponding $T$-functions,

$$
\begin{aligned}
S_{-}(x) & =\int_{0}^{\infty} \mathrm{d} y y S_{+}(y) G(y, x) \\
& =\frac{x \sqrt{4-x^{2}}\left(6-x^{2}\right)-8\left(3-x^{2}\right) \arcsin (x / 2)}{\pi x^{4}}
\end{aligned}
$$

for $x \leq 2$, and $S_{-}(x)=4\left(x^{2}-3\right) / x^{4}$ for $x>2$. Hence, the integrals in (41) do not cut off at finite separation, which was to be expected, since a constant shear cannot be uniquely assigned to an E- or B-mode, but contributes to $\left\langle|\bar{\gamma}|^{2}\right\rangle$.

\section{B-mode from source clustering}

In the previous section we have presented the decomposition of a general shear field into E/B-modes. It is usually assumed that lensing alone yields a pure E-mode shear field, so that the detection of a B-mode in the van Waerbeke et al. (2001) data (see also Pen et al. 2002) was surprising and interpreted as being due to systematic errors or a signature of intrinsic alignment of sources. Here we show that lensing indeed does generate a B-mode component of the shear if the source galaxies from which the shear is measured are clustered.

\subsection{Correlation functions and power spectra}

Define the "equivalent" surface mass density for a fixed source redshift, or comoving distance $w$,

$\kappa(\boldsymbol{\theta}, w)=\int_{0}^{w} \mathrm{~d} w^{\prime} F\left(w^{\prime}, w\right) \delta\left[f\left(w^{\prime}\right) \boldsymbol{\theta}, w^{\prime}\right]$ 
where

$F\left(w^{\prime}, w\right)=\frac{3 H_{0}^{2} \Omega_{0}}{2 c^{2}} \frac{f\left(w^{\prime}\right) f\left(w-w^{\prime}\right)}{a\left(w^{\prime}\right) f(w)} ;$

here, $H_{0}$ and $\Omega_{0}$ denote the Hubble constant and the density parameter, $w$ is the comoving distance, $f(w)$ is the comoving angular-diameter distance to comoving distance $w, \delta$ is the density contrast, and $a(w)=(1+z)^{-1}$ is the cosmic scale factor, defined such that $a=1$ today, again using the notation of BS01. Accordingly, we define the shear components

$\gamma_{\alpha}(\boldsymbol{\theta}, w)=\left(\frac{\mathcal{D}_{\alpha}}{\pi} * \kappa\right)(\boldsymbol{\theta}, w)$,

where the operator $\mathcal{D}$ is defined in (1) and (2). Then, the shear correlation function for two sources at positions $\boldsymbol{\theta}_{i}$ and distances $w_{i}$ becomes

$$
\begin{gathered}
\left\langle\gamma_{\alpha}\left(\boldsymbol{\theta}_{1}, w_{1}\right) \gamma_{\beta}\left(\boldsymbol{\theta}_{2}, w_{2}\right)\right\rangle=\left(\frac{\mathcal{D}_{\alpha} \mathcal{D}_{\beta}}{\pi^{2}}\right) * \int_{0}^{w_{1}} \mathrm{~d} w_{1}^{\prime} F\left(w_{1}^{\prime}, w_{1}\right) \\
\times \int_{0}^{w_{2}} \mathrm{~d} w_{2}^{\prime} F\left(w_{2}^{\prime}, w_{2}\right)\left\langle\delta\left[f\left(w_{1}^{\prime}\right) \boldsymbol{\theta}_{1}, w_{1}^{\prime}\right] \delta\left[f\left(w_{2}^{\prime}\right) \boldsymbol{\theta}_{2}, w_{2}^{\prime}\right]\right\rangle,
\end{gathered}
$$

where the first $\mathcal{D}$ operates on $\boldsymbol{\theta}_{1}$, and the second $\mathcal{D}$ on $\boldsymbol{\theta}_{2}$. This equation is of the form (BS 2.78), and thus we obtain, using (BS 2.82),

$$
\begin{aligned}
\left\langle\gamma_{\alpha} \gamma_{\beta}\right\rangle= & \frac{\mathcal{D}_{\alpha} \mathcal{D}_{\beta}}{\pi^{2}} * \int_{0}^{w_{1,2}} \mathrm{~d} w F\left(w, w_{1}\right) F\left(w, w_{2}\right) \\
& \times \int \frac{\mathrm{d}^{2} k}{(2 \pi)^{2}} P_{\delta}(|\boldsymbol{k}|, w) \exp \left[\operatorname{if}(w) \boldsymbol{k} \cdot\left(\boldsymbol{\theta}_{1}-\boldsymbol{\theta}_{2}\right)\right],
\end{aligned}
$$

where we have temporarily dropped the arguments of the shear correlator, $w_{1,2}=\min \left(w_{1}, w_{2}\right)$, and $P_{\delta}$ is the power spectrum of the density fluctuations which develops as a function of cosmic time (or as a function of comoving distance $w$ ). The upper limit of the integral expresses the fact that a correlated shear can only be generated by matter which is at smaller distance than both sources.

The operators $\mathcal{D}$ only act on the final term of (46) which can be evaluated using the Fourier transform of $\mathcal{D}$, as in (11),

$$
D_{\alpha} \mathcal{D}_{\beta} * \mathrm{e}^{\mathrm{i} f \boldsymbol{k} \cdot\left(\boldsymbol{\theta}_{1}-\boldsymbol{\theta}_{2}\right)}=\hat{\mathcal{D}}_{\alpha}^{*}(f \boldsymbol{k}) \hat{\mathcal{D}}_{\beta}(f \boldsymbol{k}) \mathrm{e}^{\mathrm{i} f \boldsymbol{k} \cdot\left(\boldsymbol{\theta}_{1}-\boldsymbol{\theta}_{2}\right)},
$$

so that

$$
\begin{aligned}
\left\langle\gamma_{\alpha} \gamma_{\beta}\right\rangle= & \frac{9 H_{0}^{4} \Omega_{0}^{2}}{4 c^{4}} \int_{0}^{w_{1,2}} \frac{\mathrm{d} w}{a^{2}(w)} \frac{f\left(w_{1}-w\right)}{f\left(w_{1}\right)} \frac{f\left(w_{2}-w\right)}{f\left(w_{2}\right)} \\
& \times \int \frac{\mathrm{d}^{2} \ell}{(2 \pi)^{2}} P_{\delta}\left(\frac{|\ell|}{f(w)}, w\right) \frac{\hat{\mathcal{D}}_{\alpha}^{*}(\ell) \hat{\mathcal{D}}_{\beta}(\ell)}{\pi^{2}} \mathrm{e}^{\mathrm{i} \ell \cdot\left(\boldsymbol{\theta}_{1}-\boldsymbol{\theta}_{2}\right)} .
\end{aligned}
$$

Evaluating the relevant combinations, one finds

$$
\begin{aligned}
& \left(\left\langle\gamma_{\mathrm{t}} \gamma_{\mathrm{t}}\right\rangle+\left\langle\gamma_{\times} \gamma_{\times}\right\rangle\right)\left(\theta ; w_{1}, w_{2}\right)=\frac{9 H_{0}^{4} \Omega_{0}^{2}}{4 c^{4}} \int_{0}^{w_{1,2}} \frac{\mathrm{d} w}{a^{2}(w)} \\
& \quad \times R\left(w, w_{1}\right) R\left(w, w_{2}\right) \int \frac{\mathrm{d} \ell \ell}{(2 \pi)} P_{\delta}\left(\frac{\ell}{f(w)}, w\right) J_{0}(\ell \theta)
\end{aligned}
$$

$$
\begin{aligned}
& \left(\left\langle\gamma_{\mathrm{t}} \gamma_{\mathrm{t}}\right\rangle-\left\langle\gamma_{\times} \gamma_{\times}\right\rangle\right)\left(\theta ; w_{1}, w_{2}\right)=\frac{9 H_{0}^{4} \Omega_{0}^{2}}{4 c^{4}} \int_{0}^{w_{1,2}} \frac{\mathrm{d} w}{a^{2}(w)} \\
& \times R\left(w, w_{1}\right) R\left(w, w_{2}\right) \int \frac{\mathrm{d} \ell \ell}{(2 \pi)} P_{\delta}\left(\frac{\ell}{f(w)}, w\right) J_{4}(\ell \theta)
\end{aligned}
$$

where $\theta=\left|\boldsymbol{\theta}_{1}-\boldsymbol{\theta}_{2}\right|$ and $R\left(w, w_{i}\right)=f\left(w_{i}-w\right) / f\left(w_{i}\right)$ is the ratio of the angular diameter distances of a source at $w_{i}$ seen from the distance $w \leq w_{i}$ and that seen from an observer at $w=0$.

When measuring cosmic shear from source ellipticities, the source galaxies have a broad distribution in redshift, unless information on the redshifts are available and taken into account. Hence, to calculate the observable shear correlation functions, the foregoing expressions need to be averaged over the source redshift distribution. Let $p\left(w_{1}, w_{2} ; \theta\right)$ be the probability density for comoving distances of two sources separated by an angle $\theta$ on the sky; then we have for the observable correlation functions

$\xi_{ \pm}(\theta)=\int_{0}^{w_{\mathrm{H}}} \mathrm{d} w_{1} \int_{0}^{w_{\mathrm{H}}} \mathrm{d} w_{2} p\left(w_{1}, w_{2} ; \theta\right)\left(\left\langle\gamma_{\mathrm{t}} \gamma_{\mathrm{t}}\right\rangle \pm\left\langle\gamma_{\times} \gamma_{\times}\right\rangle\right)$

and $w_{\mathrm{H}}$ is the comoving distance to the horizon. By changing the order of integration according to

$\int_{0}^{w_{\mathrm{H}}} \mathrm{d} w_{1} \int_{0}^{w_{\mathrm{H}}} \mathrm{d} w_{2} \int_{0}^{w_{1,2}} \mathrm{~d} w=\int_{0}^{w_{\mathrm{H}}} \mathrm{d} w \int_{w}^{w_{\mathrm{H}}} \mathrm{d} w_{1} \int_{w}^{w_{\mathrm{H}}} \mathrm{d} w_{2}$

we obtain

$$
\begin{aligned}
\xi_{ \pm}(\theta)= & \frac{9 H_{0}^{4} \Omega_{0}^{2}}{4 c^{4}} \int_{0}^{w_{\mathrm{H}}} \frac{\mathrm{d} w}{a^{2}(w)} \int \frac{\mathrm{d} \ell \ell}{(2 \pi)} P_{\delta}\left(\frac{\ell}{f(w)}, w\right) \\
& \times J_{0,4}(\ell \theta)\left\langle R\left(w, w_{1}\right) R\left(w, w_{2}\right)\right\rangle
\end{aligned}
$$

where the angular brackets denote the averaging of the angular-diameter distance ratios over the source distance distribution. We shall write the source redshift distribution as

$p\left(w_{1}, w_{2} ; \theta\right)=\frac{\bar{p}\left(w_{1}\right) \bar{p}\left(w_{2}\right)\left[1+\delta_{\mathrm{D}}\left(w_{1}-w_{2}\right) g\left(w_{1} ; \theta\right)\right]}{1+\omega(\theta)}$

where

$\omega(\theta)=\int_{0}^{w_{\mathrm{H}}} \mathrm{d} w \bar{p}^{2}(w) g(w ; \theta)$

is the angular correlation function of the galaxies, and $\bar{p}(w)$ describes their redshift distribution. The second term in (50) accounts for source clustering. In making this ansatz, we have accounted for the fact that redshift clustering occurs only over a very small interval in redshift over which all the other redshift-dependent functions occurring in (49) can be considered constant. Note that (50) is normalized,

$\int \mathrm{d} w_{1} \int \mathrm{d} w_{2} p\left(w_{1}, w_{2} ; \theta\right)=1$,

as required. Then, the average of the angular-diameter distance ratios becomes

$\left\langle R\left(w, w_{1}\right) R\left(w, w_{2}\right)\right\rangle=\frac{\bar{W}^{2}(w)+V(w, \theta)}{1+\omega(\theta)}$, 
where

$\bar{W}(w)=\int_{w}^{w_{\mathrm{H}}} \mathrm{d} w_{1} \bar{p}\left(w_{1}\right) \frac{f\left(w_{1}-w\right)}{f\left(w_{1}\right)}$,

$V(w, \theta)=\int_{w}^{w_{\mathrm{H}}} \mathrm{d} w_{1} \bar{p}^{2}\left(w_{1}\right)\left(\frac{f\left(w_{1}-w\right)}{f\left(w_{1}\right)}\right)^{2} g\left(w_{1} ; \theta\right)$.

The correlation of sources thus yields an average of the angular-diameter distance ratios which is not simply the square of the mean distance ratio $\bar{W}$, but contains in addition a correlated part described by $V$ and the normalization correction $1+\omega$. If the angular separation of the sources is large, the correlation in redshift is expected to be small; hence, for large separations one expects $V$ and $\omega$ to vanish. The degree of redshift correlation depends on the angular separation considered; the fact that the mean of the product of the angular diameter distance ratio (52) depends on the separation $\theta$ is the cause for a B-mode contribution to the shear correlation function!

One can check that the correlated redshift probability distribution behaves as expected in some simple cases. For example, if $w \ll c / H_{0}$ is very much smaller than the characteristic source distance $w_{0}$, one finds that

$\left\langle R\left(w, w_{1}\right) R\left(w, w_{2}\right)\right\rangle \approx 1$

in this case, the lensing strength of matter at distance $w$ is basically independent of the exact source redshift, so that source redshift clustering is irrelevant for those lens redshifts. Another case of interest occurs when the selected sources come from a very narrow distance interval, of width $\Delta w$ centered on $w_{0}$; then, (51) yields the relation $\omega(\theta) \approx g\left(w_{0} ; \theta\right) / \Delta w$, and

$\left\langle R\left(w, w_{1}\right) R\left(w, w_{2}\right)\right\rangle \approx\left[\frac{f\left(w_{0}-w\right)}{f\left(w_{0}\right)}\right]^{2} \mathrm{H}\left(w_{0}-w\right)$.

Hence, also in this case, $\langle R R\rangle$ does not depend on $\theta$, and therefore no B-mode contribution occurs - as noted before, if all sources are at the same redshift, one obtains a pure E-mode shear field.

We can now rewrite (49) in the form

$\xi_{ \pm}(\theta)=\frac{1}{1+\omega(\theta)} \int \frac{\mathrm{d} \ell \ell}{(2 \pi)} J_{0,4}(\ell \theta)\left[P_{\kappa}(\ell)+P_{\mathrm{c}}(\ell ; \theta)\right]$

where the $0(4)$ corresponds to $\xi_{+}\left(\xi_{-}\right)$,

$P_{\kappa}(\ell)=\frac{9 H_{0}^{4} \Omega_{0}^{2}}{4 c^{4}} \int_{0}^{w_{\mathrm{H}}} \frac{\mathrm{d} w}{a^{2}(w)} \bar{W}^{2}(w) P_{\delta}\left(\frac{\ell}{f(w)}, w\right)$,

and

$P_{\mathrm{c}}(\ell ; \theta)=\frac{9 H_{0}^{4} \Omega_{0}^{2}}{4 c^{4}} \int_{0}^{w_{\mathrm{H}}} \frac{\mathrm{d} w}{a^{2}(w)} V(w, \theta) P_{\delta}\left(\frac{\ell}{f(w)}, w\right)$.

The first term of (55) in the absence of source correlations (i.e., $\omega=0$ ) is the one usually derived in cosmic shear considerations; $P_{\kappa}$ is the power spectrum of the projected matter density, related to the three-dimensional power spectrum $P_{\delta}$ by a Limber-type equation (e.g., Kaiser 1992). The second term in (55) and the "normalization correction" $1+\omega$ comes about due to source correlations.

\subsection{The $E / B$-mode decomposition}

From the correlation functions (55), by writing

$$
\begin{aligned}
\frac{P_{\kappa}(\ell)+P_{\mathrm{c}}(\ell ; \theta)}{1+\omega(\theta)} & =P_{\kappa}(\ell)+\mathcal{P}(\ell ; \theta) \\
& \equiv P_{\kappa}(\ell)+\frac{P_{\mathrm{c}}(\ell ; \theta)-\omega(\theta) P_{\kappa}(\ell)}{1+\omega(\theta)},
\end{aligned}
$$

we can derive the E- and B-mode power spectra, making use of (18),

$P_{\mathrm{E}}(\ell)=P_{\kappa}(\ell)+P_{\mathrm{cE}}(\ell) ; \quad P_{\mathrm{B}}(\ell)=P_{\mathrm{cB}}(\ell) ;$

with

$$
\begin{aligned}
P_{\mathrm{cE}, \mathrm{B}}(\ell)= & \frac{1}{2} \int \mathrm{d} \theta \theta \int \mathrm{d} \ell^{\prime} \ell^{\prime} \mathcal{P}\left(\ell^{\prime} ; \theta\right) \\
& \times\left[J_{0}(\ell \theta) J_{0}\left(\ell^{\prime} \theta\right) \pm J_{4}(\ell \theta) J_{4}\left(\ell^{\prime} \theta\right)\right],
\end{aligned}
$$

where the "+" ("-")-sign corresponds to the E- (B-) mode. If the ratio containing the power spectra did not depend on $\theta, P_{\mathrm{B}}$ would vanish identically, as it should. However, this term does depend on $\theta$ due to source correlations; therefore, without using redshift information, the presence of B-modes in cosmic shear observations is unavoidable.

Using the definitions of the E- and B-mode correlation function, we obtain

$$
\begin{aligned}
& \xi_{\mathrm{E}+}(\theta)=\int \frac{\mathrm{d} \ell \ell}{(2 \pi)} P_{\kappa}(\ell) J_{0}(\ell \theta) \\
& +\int \frac{\mathrm{d} \ell \ell}{(4 \pi)}\left[\mathcal{P}(\ell ; \theta)\left[J_{0}(\ell \theta)+J_{4}(\ell \theta)\right]\right. \\
& \left.+\int_{\theta}^{\infty} \mathrm{d} \vartheta \vartheta \mathcal{P}(\ell ; \vartheta) J_{4}(\ell \vartheta)\left(\frac{4}{\vartheta^{2}}-\frac{12 \theta^{2}}{\vartheta^{4}}\right)\right], \\
& \xi_{\mathrm{B}+}(\theta)=\int \frac{\mathrm{d} \ell \ell}{(4 \pi)}\left[\mathcal{P}(\ell ; \theta)\left[J_{0}(\ell \theta)-J_{4}(\ell \theta)\right]\right. \\
& \left.-\int_{\theta}^{\infty} \mathrm{d} \vartheta \vartheta \mathcal{P}(\ell ; \vartheta) J_{4}(\ell \vartheta)\left(\frac{4}{\vartheta^{2}}-\frac{12 \theta^{2}}{\vartheta^{4}}\right)\right], \\
& \xi_{\mathrm{E}-}(\theta)=\int \frac{\mathrm{d} \ell \ell}{(2 \pi)} P_{\kappa}(\ell) J_{4}(\ell \theta) \\
& +\int \frac{\mathrm{d} \ell \ell}{(4 \pi)}\left[\mathcal{P}(\ell ; \theta)\left[J_{0}(\ell \theta)+J_{4}(\ell \theta)\right]\right. \\
& \left.+\int_{0}^{\theta} \mathrm{d} \vartheta \vartheta \mathcal{P}(\ell ; \vartheta) J_{0}(\ell \vartheta)\left(\frac{4}{\theta^{2}}-\frac{12 \vartheta^{2}}{\theta^{4}}\right)\right], \\
& \xi_{\mathrm{B}-}(\theta)=\int \frac{\mathrm{d} \ell \ell}{(4 \pi)}\left[\mathcal{P}(\ell ; \theta)\left[J_{0}(\ell \theta)-J_{4}(\ell \theta)\right]\right. \\
& \left.+\int_{0}^{\theta} \mathrm{d} \vartheta \vartheta \mathcal{P}(\ell ; \vartheta) J_{0}(\ell \vartheta)\left(\frac{4}{\theta^{2}}-\frac{12 \vartheta^{2}}{\theta^{4}}\right)\right] .
\end{aligned}
$$

\section{Relative strength of the B-mode}

Whereas the presence of a B-mode, and an additional contribution to the E-mode due to source clustering must occur, one needs to estimate the relative amplitude of this 
effect as compared to the "usual" cosmic shear strength described by $P_{\kappa}$. This estimate requires a model for the source clustering, i.e., a model for the function $g(w ; \theta) . g$ can be related to the three-dimensional correlation function $\xi_{\mathrm{gg}}$ of galaxies,

$g(w ; \theta)=\int_{-\infty}^{\infty} \mathrm{d}(\Delta w) \xi_{\text {gg }}\left(\sqrt{(\Delta w)^{2}+f^{2}(w) \theta^{2}}\right)$.

If we assume that the correlation function behaves like a power-law, $\xi_{\mathrm{gg}}(r)=\left[r / r_{0}(w)\right]^{-\gamma}$, where $r_{0}(w)$ is the comoving correlation length, and $\gamma \simeq 1.7$, then

$g(w ; \theta)=C r_{0}(w)\left(\frac{f(w) \theta}{r_{0}(w)}\right)^{1-\gamma}$,

where $C=\sqrt{\pi} \Gamma([\gamma-1] / 2) / \Gamma(\gamma / 2)$, and $\Gamma(x)$ is the Gamma function. This yields for the angular two-point correlation function

$\omega(\theta)=\theta^{(1-\gamma)} C \int_{0}^{w_{\mathrm{H}}} \mathrm{d} w \bar{p}^{2}(w) r_{0}(w)\left(\frac{f(w)}{r_{0}(w)}\right)^{1-\gamma}$.

A useful parameterization of $\omega(\theta)$ is $\omega=A\left(1^{\prime}\right)\left(\theta / 1^{\prime}\right)^{(1-\gamma)}$. Fixing $\gamma=1.7$, one obtains a relation between the correlation length $r_{0}(w)$ and the redshift distribution $\bar{p}(w)$,

$\int_{0}^{w_{\mathrm{H}}} \mathrm{d} w \bar{p}^{2}(w) r_{0}(w)\left(\frac{f(w)}{r_{0}(w)}\right)^{-0.7}=1.65 \times 10^{-5} \frac{A\left(1^{\prime}\right)}{0.02}$,

where the fiducial value of $A\left(1^{\prime}\right)$ was taken from McCracken et al. (2001). Note that in McCracken et al. (2001), essentially the same data set has been used as in the cosmic shear analysis of van Waerbeke et al. (2001); in particular, the depth of the data are the same. The above-quoted value for the angular clustering strength at $1^{\prime}$ corresponds to the faintest flux threshold considered in McCracken et al., which is very similar to the flux limit employed in the cosmic shear analysis. It must be mentioned, however, that the galaxies used in the cosmic shear analysis do not form a truly flux-limited sample, since additional cuts are used, e.g. a size cut. Hence, a precise estimate of the angular correlation function of those galaxies which were used for the cosmic shear analysis cannot be given.

For this reason, we shall assume the power-law dependence of $\omega(\theta)$ as given above; in addition, we will make the simplifying assumption that the comoving clustering length $r_{0}(w)$ is independent of distance $w$; this assumption is not too critical, since the function $\bar{p}^{2}(w)$ is relatively well peaked and therefore large $w$-variations of the correlation length are not probed. Then, (68) determines this constant comoving correlation length $r_{0}$. We obtain in this case

$g(w ; \theta)=\omega(\theta) \frac{[f(w)]^{1-\gamma}}{\int \mathrm{d} w^{\prime} \bar{p}^{2}\left(w^{\prime}\right)[f(w)]^{1-\gamma}}$.

The power-law dependence of $g$ on $\theta$ implies that $P_{\mathrm{c}}(\ell ; \theta)$ also behaves like $\theta^{1-\gamma}$. Since the angular correlation function is small compared to unity, even on scales of a few

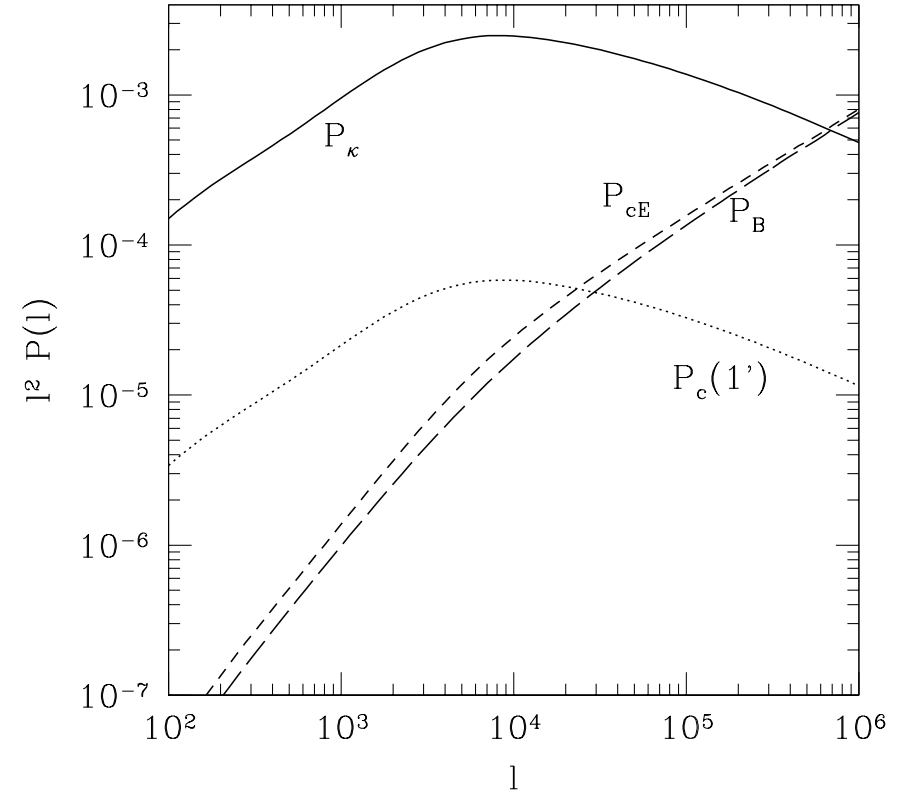

Fig. 2. Dimensionless power spectra $\ell^{2} P(\ell)$, as a function of of wavenumber $\ell$. The solid curve corresponds to the power spectrum $\ell^{2} P_{\kappa}(\ell)$ that is the "standard" power spectrum of the projected mass density. The dotted curve displays $\ell^{2} P_{\mathrm{c}}\left(\ell, 1^{\prime}\right)$, and the two dashed curves correspond to the E- and B-mode power caused by the source clustering. Here, a $\Lambda \mathrm{CDM}$ model was used, with shape parameter $\Gamma=0.21$, normalization $\sigma_{8}=$ 1 , and the source redshift distribution is characterized by $z_{0}=$ 1 , yielding $\langle z\rangle \approx 1.5$. Other parameters for the model used here are mentioned in the text.

arcseconds, we shall neglect $\omega(\theta)$ in the denominator of the integrand in (60); this greatly simplifies the calculation of the power spectra due to source clustering, since the $\theta$-integration can then be carried out first, making use of Eqs. $(11.4 .33,34)$ of AS.

In order to make further progress, we need to assume a redshift distribution for the sources from which the shear is measured. We employ the form (Brainerd et al. 1996)

$\bar{p}_{z}(z) \propto z^{2} \exp \left[-\left(\frac{z}{z_{0}}\right)^{\beta}\right]$

and shall consider $\beta=3 / 2$ in the following, yielding a mean redshift of $\langle z\rangle \approx 1.5 z_{0}$.

In Fig. 2 we show an example of the various power spectra considered here; all power spectra are multiplied by $\ell^{2}$ to obtain dimensionless quantities. For this figure, we employed a standard $\Lambda$ CDM model with $\Omega_{\Lambda}=0.7$ and $\Omega_{0}=0.3$ and normalization $\sigma_{8}=1$. Sources are distributed in redshift according to the foregoing prescription, with $z_{0}=1$. The amplitude of the angular correlation function of galaxies was chosen to be $A\left(1^{\prime}\right)=0.02$, and the slope of $\gamma=1.7$ for the three-dimensional correlation function was used; the corresponding correlation length in this case is $r_{0} \approx 4.7 h^{-1} \mathrm{Mpc}$. To calculate the threedimensional power spectrum and its redshift evolution, we used the Peacock \& Dodds (1996) prescription for the non-linear evolution of $P_{\delta}(k, w)$. 
The power spectrum of the projected mass density, $P_{\kappa}(\ell)$, is the same as that in SvWJK, except for a slightly different choice of the cosmological parameters. The spectrum $P_{\mathrm{c}}\left(\ell ; 1^{\prime}\right)$ is very much smaller than $P_{\kappa}(\ell)$, as expected from the smallness of the amplitude of the angular correlation function; in fact, the ratio $P_{\mathrm{c}}\left(\ell ; 1^{\prime}\right) / P_{\kappa}(\ell)$ is nearly constant at a value of approximately $(1+B) A\left(1^{\prime}\right)$, with $B \approx 1.2$ for this choice of the parameters.

The behavior of the power spectra which arise from source clustering, $P_{\mathrm{cE}}$ and $P_{\mathrm{B}} \equiv P_{\mathrm{cB}}$, as a function of $\ell$ is quite different. First, both of these spectra are very similar, which is due to the fact that the $J_{4}$-term in (60) is much smaller than the $J_{0}$-term. Second, although both of these spectra are small on large angular scales, i.e. at small $\ell$, their relative value increases strongly for smaller angular scales. Hence, as expected, the relative importance of source clustering increases for larger $\ell$. What is surprising, though, is that these power spectra have the same amplitude as $P_{\kappa}$ at a value of $\ell \sim 6.7 \times 10^{5}$, corresponding to an angular scale of $\theta=2 \pi / \ell \sim 2^{\prime \prime}$, and the relative contribution of the B-mode amounts to about $2 \%$ at an angular scale of $1^{\prime}$. It should be noted here that cosmic shear has already been measured on scales below $1^{\prime}$; therefore, source clustering gives rise to a B-mode component in cosmic shear which is observable.

We shall now consider the behavior of $P_{\mathrm{B}}(\ell)$ for large values of $\ell$. The aforementioned properties of $P_{\mathrm{c}}(\ell ; \theta)$ can be summarized as

$P_{\mathrm{c}}(\ell ; \theta) \approx(1+B) A\left(1^{\prime}\right) P_{\kappa}(\ell)\left(\frac{\theta}{1^{\prime}}\right)^{1-\gamma}$.

Inserting this result into (60), neglecting the $J_{4}$-terms and considering the limit $\ell \rightarrow \infty$ yields

$\ell^{2} P_{\mathrm{B}}(\ell) \approx a_{1} B A\left(1^{\prime}\right)\left(\ell 1^{\prime}\right)^{\gamma-1} \int \mathrm{d} \ell \ell P_{\kappa}(\ell)$,

where $a_{1}=2^{1-\gamma} \Gamma[(3-\gamma) / 2] / \Gamma[(\gamma-1) / 2] \approx 0.335$ for $\gamma=1.7$. Hence, $P_{\mathrm{B}} \propto \ell^{\gamma-3}$ at large $\ell$. To obtain an approximate value for the integral in the preceding equation, we shall describe the power spectrum $P_{\kappa}$ by a simple function,

$\ell^{2} P_{\kappa}(\ell) \sim B_{\mathrm{m}} \frac{\left(\ell / \ell_{\mathrm{m}}\right)^{\alpha}}{\left[1+\left(\ell / \ell_{\mathrm{m}}\right)^{2}\right]^{\beta}}$

with $B_{\mathrm{m}} \sim 3.7 \times 10^{-3}, \alpha \sim 0.7, \beta \sim 0.6$, and $\ell_{\mathrm{m}} \sim 7 \times 10^{3}$. Using $A\left(1^{\prime}\right)=0.02$, this then yields

$\ell^{2} P_{\mathrm{B}}(\ell) \approx 1.5 \times 10^{-4}\left(\frac{\ell}{10^{5}}\right)^{0.7}$,

which is a reasonably good description of the result in Fig. 2 for large $\ell$. Furthermore, we can obtain the ratio $P_{\mathrm{B}} / P_{\kappa}$ in the limit of large $\ell \gg \ell_{\mathrm{m}}$, which yields

$\frac{P_{\mathrm{B}}(\ell)}{P_{\kappa}(\ell)} \approx\left(\frac{\ell}{4.9 \times 10^{5}}\right)^{1.2}$

and roughly predicts the correct crossing point between these two power spectra seen in Fig. 2.

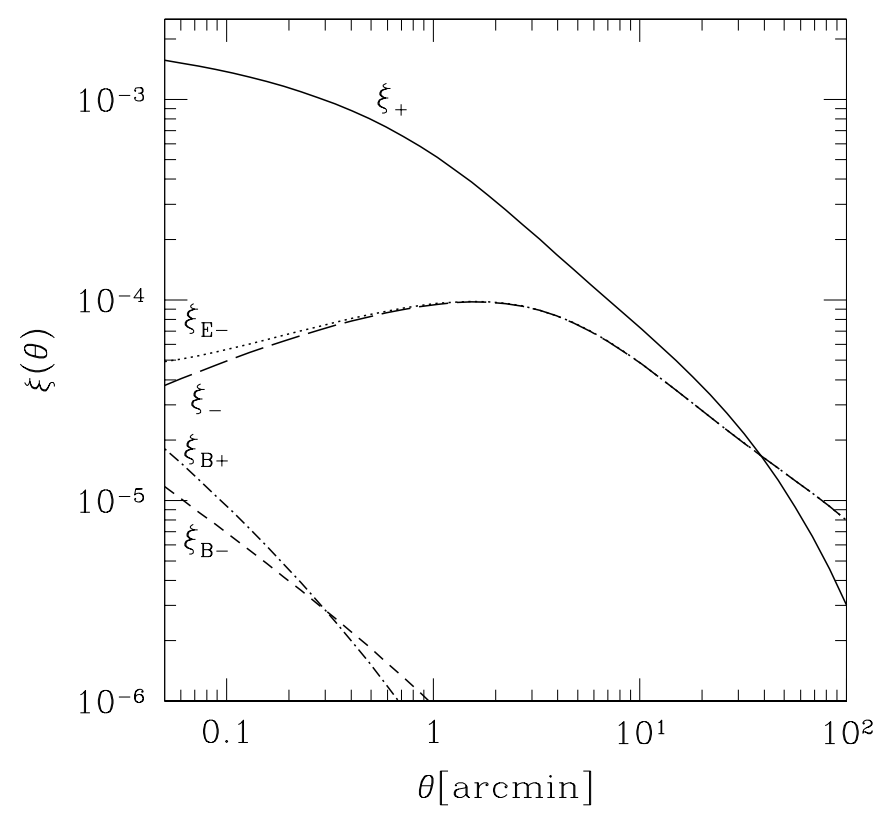

Fig. 3. For the same model as in Fig. 2, several correlation functions are plotted. The solid line shows $\xi_{+}(\theta)$; in fact, the correlation function $\xi_{\mathrm{E}+}$ cannot be distinguished from $\xi_{+}$on the scale of this figure; their fractional difference is less than $1 \%$, even on the smallest scale shown. The two B-mode correlation functions are shown as well as $\xi_{-}$and $\xi_{\mathrm{E}-}$. Note that the difference between the latter two is larger than that of the corresponding "+"-correlation functions.

In Fig. 3 we have plotted several correlation functions; they have been calculated from the power spectra plotted in Fig. 2 by using (19). The first point to note is that $\xi_{\mathrm{E}+}$ differs from $\xi_{+}$by less than $1 \%$ for angular scales larger than $3^{\prime \prime}$; hence, the relative contribution caused by the source correlation is even smaller than that seen in the power spectra. This is due to the fact that the correlation function is a filtered version of the power spectra, however with a very broad filter. This implies that even at small $\theta$ scales, the correlation function is not dominated by large values of $\ell$, where the contribution from source clustering is largest, but low values of $\ell$ contribute significantly. The influence of source clustering on the "-" modes is larger, since the filtering function for those are narrower (i.e., $J_{4}(x)$ is a more localized function that $\left.J_{0}(x)\right)$, and $\xi_{\mathrm{E}-}$ differs from $\xi_{-}$appreciably on scales below about $1^{\prime}$.

Finally, in Fig. 4 we have plotted the aperture measures. On scales below about $1^{\prime}$, the dispersion of $M_{\perp}$ is larger than about $1 \%$ of that of $M_{\mathrm{ap}}$. Hence, the ratio of these E- and B-mode aperture measures are very similar to that of the corresponding power spectra.

The fact that $P_{\mathrm{cE}}$ and $P_{\mathrm{B}}$ are very similar in amplitude means that by measuring $P_{\mathrm{B}}$, one can make an approximate correction of $P_{\mathrm{E}}$, obtaining a value close to $P_{\kappa}$ by subtracting $P_{\mathrm{B}}$ from $P_{\mathrm{E}}$. Owing to the relative amplitude of these correlation-induced powers, such a correction may be needed in future high-precision measurements of the cosmic shear. 


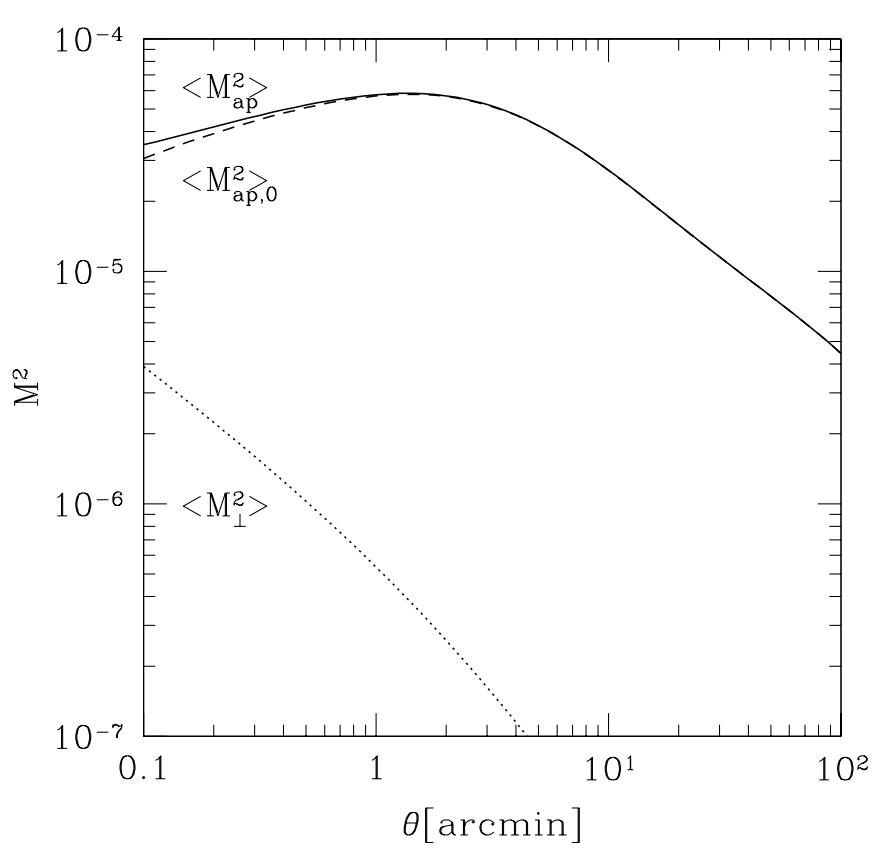

Fig. 4. Aperture measures, for the same model as used in Fig. 2. Shown here is the dispersion of the aperture mass, $\left\langle M_{\mathrm{ap}}^{2}\right\rangle$, the corresponding function in the absence of source correlations (noted by the subscript " 0 ") and $\left\langle M_{\perp}^{2}\right\rangle$, which is the aperture measure for the B-mode. As expected from the power spectra shown in Fig. 2, and the fact that the aperture measures are a filtered version of the power spectra with a very narrow filter function, the B-mode aperture measure is considerably smaller than $M_{\text {ap }}$ itself.

\section{Discussion}

We have shown that the clustering of galaxies from which the shear is measured leads to the presence of a B-mode in the cosmic shear field, in addition to providing an additional component to the E-mode. The reason for this effect in essence is the angular separation-dependent redshift correlation of galaxies, which causes the mean of the product of the angular-diameter distance ratio along two lines-of-sight not to factorize, but to depend on $\theta$. For a fiducial model considered in detail, the B-mode contribution amounts to more than $\sim 2 \%$ on angular scales below $1^{\prime}$ (or $\ell \gtrsim 2.16 \times 10^{4}$ ), and its relative importance quickly rises towards smaller angles. On substantially larger angular scales, however, the B-mode contribution is small. Furthermore, the additional E-mode contribution is very similar in size to the B-mode power, which will allow an approximate correction of the measured E-mode for this additional term.

From an observational point-of-view, the most easily accessible quantities are the shear correlation functions $\xi_{ \pm}$, as one can easily deal with gaps in the data field. In Sect. 2 we have given explicit relations regarding how other two-point statistics of the shear can be calculated in terms of the shear correlation function. The finite support of the functions $T_{ \pm}$indicates that the aperture measures are more easily obtained from observational data than either the E- and B-mode correlation functions, or the E- and B-mode shear dispersions. Therefore, the aperture measures are the preferred method to check for the presence of a B-mode contribution in the shear data.

We have varied some of the model parameters; in particular, we have considered the case of lower mean source redshift (corresponding to a brighter flux threshold), and simultaneously increasing $A\left(1^{\prime}\right)$, such that the clustering length $r_{0}$ stays about the same. In this case we found a very similar ratio between the B- and E-mode power spectra as for the example considered in Sect. 4. We consider it unlikely that the observed B-mode in the present day data sets is due to the source clustering effect. The B-mode found in van Waerbeke et al. (2001) and Pen et al. (2002) can actually be used to search quantitatively for residual systematics. Its detection in van Waerbeke et al. (2001) was done by obtaining $M_{\text {ap }}$ and $M_{\perp}$ by laying down a grid of circular apertures on the data field. A more accurate measurement of $\left\langle M_{\mathrm{ap}}^{2}\right\rangle$ and $\left\langle M_{\perp}^{2}\right\rangle$ has been obtained from the same data by Pen et al. (2002), by calculating them from the observed correlation functions $\xi_{ \pm}$, as in (32). In fact a subsequent analysis revealed that the B-mode measured in these data were essentially residual systematics caused by an overcorrection of the PSF, and can be corrected for (van Waerbeke et al. 2002). In this latter analysis, no significant B-modes are detected at small angular scales, but on scales above $\sim 10^{\prime}$, slightly significant values of $M_{\perp}$ are detected; the effect discussed in this paper can certainly not account for them.

The effect considered here seems to have been overlooked hitherto. Bernardeau (1998) considered the effects of source clustering on cosmic shear statistics and concluded that this source clustering can strongly affect the skewness and kurtosis of the cosmic shear, but to first order leaves the shear dispersion (and thus the power spectrum) unaffected. Hamana et al. (2002) studied this effect with ray tracing simulations, again concentrating on the skewness. Most of the other ray tracing simulations of weak lensing (e.g., van Waerbeke et al. 1999; Jain et al. 2000) assumed all sources to be at the same redshift, in which case the additional power discussed here does not occur. Lombardi et al. (2002) calculated the effect of source clustering on the noise of weak lensing mass maps, showing that it can provide a significant noise contribution in the inner regions of clusters.

It must be pointed out that the effect considered here is unrelated to other lensing effects which in principle could generate a B-mode, such as lens-lens coupling or the break-down of the Born approximation (see Bernardeau et al. 1997 and SvWJK for a discussion of these two effects on the skewness). Numerical estimates (e.g., Jain et al. 2000) show that these latter two effects are very weak. Bertin \& Lombardi (2001) considered the situation of lensing by two mass concentrations along the line-of-sight, where a B-mode is generated by a strong lens-lens coupling, but the fraction of lines-of-sight where this occurs is tiny. Another effect which could in principle generate a 
B-mode from lensing is the fact that the observable is not the shear itself, but the reduced shear (Schneider \& Seitz 1995). In the appendix we show that this effect is indeed negligible.

Like the intrinsic alignment of galaxies, which can yield a spurious contribution to the measured cosmic shear, the source clustering effect can in principle be avoided if redshift estimates of the source galaxies are available. In that case, by estimating the shear correlation function, pairs of galaxies with a large likelihood to be at the same distance can be neglected. In contrast to the intrinsic correlation of galaxies, the B-mode from source clustering appears to be fairly insensitive to the redshift distribution of the source galaxies, provided the clustering length is kept fixed.

Acknowledgements. We thank L. J. King for useful comments on the manuscript. This work was supported by the TMR Network "Gravitational Lensing: New Constraints on Cosmology and the Distribution of Dark Matter" of the EC under contract No. ERBFMRX-CT97-0172 and by the German Ministry for Science and Education (BMBF) through the DLR under the project 50 OR 0106.

\section{Appendix A: B-mode from reduced shear?}

The shear is not directly an observable, but is estimated from the image ellipticities of distant galaxies. The expectation value of the image ellipticity, however, is not the shear, but the reduced shear $g=\gamma /(1-\kappa)$. Hence, the correlation of the observed ellipticities is the correlation of the reduced shear, not the shear itself. In cosmic shear, $|\kappa| \ll 1$ nearly everywhere, and so the difference between shear and reduced shear shall not play a big role. However, at least a priori, this effect cannot be neglected, as seen from the following argument:

The skewness $S_{3}=\left\langle X^{3}\right\rangle /\left\langle X^{2}\right\rangle^{2}$, where $X$ is a measure of shear (such as $M_{\text {ap }}$, or the reconstructed $\kappa$ ) has been calculated by van Waerbeke et al. (2001) to be of order a few hundred. On a scale of about one arcminute, $\left\langle X^{2}\right\rangle \sim 5 \times 10^{-4}$, so that $\left\langle X^{3}\right\rangle \sim 0.1\left\langle X^{2}\right\rangle$, taking $S_{3} \sim 200$ for the top-hat smoothed $\kappa$. The difference between the correlation functions involving $g$ and those involving $\gamma$ is in principle of the same order-of-magnitude as $\left\langle X^{3}\right\rangle$ and thus can be present at the level of a few percent, and there is no reason why it should not contain a B-mode contribution.

We define the correlation functions

$\xi_{ \pm}^{g}(\theta)=\left\langle g_{\mathrm{t}}(\mathbf{0}) g_{\mathrm{t}}(\boldsymbol{\theta})\right\rangle \pm\left\langle g_{\times}(\mathbf{0}) g_{\times}(\boldsymbol{\theta})\right\rangle$

and choose $\boldsymbol{\theta}=(\theta, 0)$, so that $g_{\mathrm{t}}=-g_{1}, g_{\times}=-g_{2}$. Using the approximation $g \approx \gamma(1+\kappa)$, valid for $|\kappa| \ll 1$, we obtain

$\xi_{ \pm}^{g}(\theta)=\xi_{ \pm}(\theta)+\Delta \xi_{ \pm}(\theta)$,

where

$$
\begin{aligned}
\Delta \xi_{ \pm}(\theta)= & \left\langle\gamma_{1}(\mathbf{0}) \gamma_{1}(\boldsymbol{\theta})[\kappa(\mathbf{0})+\kappa(\boldsymbol{\theta})]\right\rangle \\
& \pm\left\langle\gamma_{2}(\mathbf{0}) \gamma_{2}(\boldsymbol{\theta})[\kappa(\mathbf{0})+\kappa(\boldsymbol{\theta})]\right\rangle .
\end{aligned}
$$

Replacing the shear and convergence by their Fourier transforms, this becomes

$$
\begin{aligned}
\Delta \xi_{ \pm}(\theta)= & \int \frac{\mathrm{d}^{2} \ell_{1}}{(2 \pi)^{2}} \int \frac{\mathrm{d}^{2} \ell_{2}}{(2 \pi)^{2}} \int \frac{\mathrm{d}^{2} \ell_{3}}{(2 \pi)^{2}}\left\langle\hat{\kappa}\left(\boldsymbol{\ell}_{1}\right) \hat{\kappa}\left(\boldsymbol{\ell}_{2}\right) \hat{\kappa}\left(\boldsymbol{\ell}_{3}\right)\right\rangle \\
& \times\left(\mathrm{e}^{-\mathrm{i} \ell_{2} \cdot \boldsymbol{\theta}}+\mathrm{e}^{-\mathrm{i}\left(\ell_{2}+\ell_{3}\right) \cdot \boldsymbol{\theta}}\right) \cos \left[2\left(\beta_{1} \mp \beta_{2}\right)\right],(\mathrm{A}, \boldsymbol{4})
\end{aligned}
$$

where, as before, $\beta_{i}$ is the polar angle of $\ell_{i}$. The triple correlator vanishes unless the sum of the wave-vectors equals zero; one defines the bispectrum by

$$
\left\langle\hat{\kappa}\left(\boldsymbol{\ell}_{1}\right) \hat{\kappa}\left(\boldsymbol{\ell}_{2}\right) \hat{\kappa}\left(\boldsymbol{\ell}_{3}\right)\right\rangle=(2 \pi)^{2} \delta\left(\boldsymbol{\ell}_{1}+\boldsymbol{\ell}_{2}+\boldsymbol{\ell}_{3}\right) b\left(\boldsymbol{\ell}_{1}, \boldsymbol{\ell}_{2}, \boldsymbol{\ell}_{3}\right) .
$$

Performing the $\ell_{3}$-integration in (A.4) yields

$$
\begin{aligned}
\Delta \xi_{ \pm}(\theta)= & \int \frac{\mathrm{d}^{2} \ell_{1}}{(2 \pi)^{2}} \int \frac{\mathrm{d}^{2} \ell_{2}}{(2 \pi)^{2}} b\left(\boldsymbol{\ell}_{1}, \boldsymbol{\ell}_{2},-\boldsymbol{\ell}_{1}-\boldsymbol{\ell}_{2}\right) \\
& \times\left(\mathrm{e}^{-\mathrm{i} \ell_{2} \cdot \boldsymbol{\theta}}+\mathrm{e}^{\mathrm{i} \ell_{1} \cdot \boldsymbol{\theta}}\right) \cos \left[2\left(\beta_{1} \mp \beta_{2}\right)\right] .
\end{aligned}
$$

The function $b\left(\ell_{1}, \ell_{2},-\ell_{1}-\ell_{2}\right)$ has three independent arguments, namely the moduli $\ell_{1}$ and $\ell_{2}$, and the angle $\phi=\beta_{1}-\beta_{2}$ between the two $\ell$-vectors. We therefore write $b\left(\ell_{1}, \ell_{2},-\ell_{1}-\ell_{2}\right)=\tilde{b}\left(\ell_{1}, \ell_{2}, \phi\right)$, make use of the symmetry in the integrand of (A.6), and replace the $\beta_{1}$-integration by one over $\phi$ :

$$
\begin{aligned}
\Delta \xi_{+}(\theta)= & 2 \int_{0}^{\infty} \frac{\mathrm{d} \ell_{1} \ell_{1}}{(2 \pi)^{2}} \int_{0}^{\infty} \frac{\mathrm{d} \ell_{2} \ell_{2}}{(2 \pi)^{2}} \int_{0}^{2 \pi} \mathrm{d} \phi \tilde{b}\left(\ell_{1}, \ell_{2}, \phi\right) \\
& \times \int_{0}^{2 \pi} \mathrm{d} \beta_{2} \mathrm{e}^{-\mathrm{i} \ell_{2} \theta \cos \beta_{2}} \cos (2 \phi) \\
= & \frac{1}{\pi} \int_{0}^{\infty} \frac{\mathrm{d} \ell_{1} \ell_{1}}{(2 \pi)} J_{0}\left(\ell_{1} \theta\right) \int_{0}^{\infty} \frac{\mathrm{d} \ell_{2} \ell_{2}}{(2 \pi)} \\
& \times \int_{0}^{2 \pi} \mathrm{d} \phi \cos (2 \phi) \tilde{b}\left(\ell_{1}, \ell_{2}, \phi\right)
\end{aligned}
$$

analogously, one obtains

$$
\begin{aligned}
\Delta \xi_{-}(\theta)= & \frac{1}{\pi} \int_{0}^{\infty} \frac{\mathrm{d} \ell_{1} \ell_{1}}{(2 \pi)} J_{4}\left(\ell_{1} \theta\right) \int_{0}^{\infty} \frac{\mathrm{d} \ell_{2} \ell_{2}}{(2 \pi)} \\
& \times \int_{0}^{2 \pi} \mathrm{d} \phi \cos (2 \phi) \tilde{b}\left(\ell_{1}, \ell_{2}, \phi\right) .
\end{aligned}
$$

Inserting these expressions into (18) and making use of (17), one immediately sees that the reduced shear does not yield any B-mode contribution, and that the correlation functions for the reduced shear are

$\xi_{ \pm}^{g}(\theta)=\int \frac{\mathrm{d} \ell \ell}{2 \pi} J_{0,4}(\ell \theta)\left[P_{\kappa}(\ell)+P^{(3)}(\ell)\right]$,

where

$P^{(3)}(\ell)=2 \int \frac{\mathrm{d}^{2} \ell^{\prime}}{(2 \pi)^{2}}\left[\frac{2\left(\boldsymbol{\ell} \cdot \boldsymbol{\ell}^{\prime}\right)^{2}}{|\boldsymbol{\ell}|^{2}\left|\ell^{\prime}\right|^{2}}-1\right] b\left(\boldsymbol{\ell}, \boldsymbol{\ell}^{\prime},-\boldsymbol{\ell}-\boldsymbol{\ell}^{\prime}\right) .($

Thus, considering the reduced shear yields an additional E-mode power to the one obtained from considering the shear itself. 


\section{References}

Abramowitz, M., \& Stegun, I. A. 1965, Handbook of mathematical functions (Dover, New York) (AS)

Bacon, D. J., Refregier, A. R., \& Ellis, R. S. 2000, MNRAS, 318,625

Bartelmann, M., \& Schneider, P. 1999, A\&A, 345, 17

Bartelmann, M., \& Schneider, P. 2001, Phys. Rep., 340, 291 (BS01)

Bernardeau, F. 1998, A\&A, 338, 375

Bernardeau, F., Van Waerbeke, L., \& Mellier, Y. 1997, A\&A, 322,1

Bertin, G., \& Lombardi, M. 2001, ApJ, 546, 47

Blandford, R. D., Saust, A. B., Brainerd, T. G., \& Villumsen, J. V. 1991, MNRAS, 251, 600

Brainerd, T. G., Blandford, R. D., \& Smail, I. 1996, ApJ, 466, 623

Catelan, P., Kamionkowski, M., \& Blandford, R. D. 2001, MNRAS, 320, L7

Crittenden, R. G., Natarajan, P., Pen, U.-L., \& Theuns, T. 2001a, ApJ, 559, 552

Crittenden, R. G., Natarajan, P., Pen, U.-L. \& Theuns, T. 2001b, ApJ, 568, 20

Croft, R. A. C., \& Metzler, C. A. 2001, ApJ, 545, 561

Gradshteyn, I. S., \& Ryzhik, I. M. 1980, Table of integrals, series and products (Academic Press)

Gunn, J. E. 1967, ApJ, 150, 737

Hämmerle, H., Miralles, J.-M., Schneider, P., et al. 2002, A\&A, 385,743

Hamana, T., Colombi, S. T., Thion, A., et al. 2002, MNRAS, 330,365

Heavens, A. F., Refregier, A., \& Heymans, C. E. C. 2000, MNRAS, 319, 649

Jain, B., \& Seljak, U. 1997, ApJ, 484, 560

Jain, B., Seljak, U., \& White, S. D. M. 2000, ApJ, 530, 547

Kaiser, N. 1992, ApJ, 388, 272

Kaiser, N. 1998, ApJ, 498, 26
Kaiser, N., \& Squires, G. 1993, ApJ, 404, 441

Kaiser, N., Wilson, G., \& Luppino, G. 2000 [astro-ph/0003338]

Kantowski, R. 1969, ApJ, 155, 89

Lombardi, M., \& Bertin, G. 1998, A\&A, 335, 1

Lombardi, M., Schneider, P., \& Morales-Morino, C. 2002, A\&A, 382, 769

Maoli, R., van Waerbeke, L., Mellier, Y., et al. 2001, A\&A, 368,766

McCracken, H. J., Le Fevre, O., Brodwin, M., et al. 2001, A\&A, 376,756

Mellier, Y. 1999, ARA\&A, 37, 127

Miralda-Escudé, J. 1991, ApJ, 380, 1

Peacock, J. A., \& Dodds, S. J. 1996, MNRAS, 280, L19

Pen, U.-L., van Waerbeke, L., \& Mellier, Y. 2002, ApJ, 567, 31

Reblinsky, K., Kruse, G., Jain, B., \& Schneider, P. 1999, A\&A, 351, 815

Rhodes, J., Refregier, A., \& Groth, E. J. 2001, ApJ, 552, L85

Schneider, P. 1996, MNRAS, 283, 837

Schneider, P., \& Seitz, C. 1995, A\&A, 294, 411

Schneider, P., van Waerbeke, L., Jain, B., \& Kruse, G. 1998, MNRAS, 296, 873 (SvWJK)

Seitz, S., \& Schneider, P. 1996, A\&A, 305, 383

Seitz, S., \& Schneider, P. 2001, A\&A, 374, 740

van Waerbeke, L. 2000, MNRAS, 313, 524

van Waerbeke, L., Bernardeau, F., \& Mellier, Y. 1999, A\&A, 243, 15

Van Waerbeke, L., Mellier, Y., Erben, T., et al. 2000, A\&A, 358, 30

Van Waerbeke, L., Mellier, Y., Radovich, M., et al. 2001, A\&A, 374, 757

van Waerbeke, L., Mellier, Y., Pello, R., et al. 2002 [astro-ph/0202503]

White, M., \& Hu, W. 2000, ApJ, 537, 1

Wittman, D. M., Tyson, J. A., Kirkman, D., Dell'Antonio, I., \& Bernstein, G. 2000, Nature, 405, 143 